\title{
A Hybrid Optimized Weighted Minimum Spanning Tree for the Shortest Intrapath Selection in Wireless Sensor Network
}

\author{
Matheswaran Saravanan ${ }^{1}$ and Muthusamy Madheswaran ${ }^{2}$ \\ ${ }^{1}$ Department of Computer Science and Engineering, VMKV Engineering College, Salem, Tamil Nadu 636308, India \\ ${ }^{2}$ Department of Electronics and Communication Engineering, Mahendra Engineering College, Namakkal, Tamil Nadu 637503, India \\ Correspondence should be addressed to Matheswaran Saravanan; saravanan.vmkv@rediffmail.com and Muthusamy Mad- \\ heswaran; madheswaran.dr@gmail.com
}

Received 11 April 2014; Revised 22 June 2014; Accepted 22 June 2014; Published 22 July 2014

Academic Editor: Jer-Guang Hsieh

Copyright (C) 2014 M. Saravanan and M. Madheswaran. This is an open access article distributed under the Creative Commons Attribution License, which permits unrestricted use, distribution, and reproduction in any medium, provided the original work is properly cited.

\begin{abstract}
Wireless sensor network (WSN) consists of sensor nodes that need energy efficient routing techniques as they have limited battery power, computing, and storage resources. WSN routing protocols should enable reliable multihop communication with energy constraints. Clustering is an effective way to reduce overheads and when this is aided by effective resource allocation, it results in reduced energy consumption. In this work, a novel hybrid evolutionary algorithm called Bee Algorithm-Simulated Annealing Weighted Minimal Spanning Tree (BASA-WMST) routing is proposed in which randomly deployed sensor nodes are split into the best possible number of independent clusters with cluster head and optimal route. The former gathers data from sensors belonging to the cluster, forwarding them to the sink. The shortest intrapath selection for the cluster is selected using Weighted Minimum Spanning Tree (WMST). The proposed algorithm computes the distance-based Minimum Spanning Tree (MST) of the weighted graph for the multihop network. The weights are dynamically changed based on the energy level of each sensor during route selection and optimized using the proposed bee algorithm simulated annealing algorithm.
\end{abstract}

\section{Introduction}

Wireless sensor network (WSN) is a cooperative collection of sensor nodes, each having processing capability. Routing in WSN is different from conventional fixed network routing by several ways. WSNs are infrastructureless, have unreliable wireless links, contain sensor nodes that might fail, and its routing protocols face rigorous energy saving requirements [1]. WSN is a distributed real-time system and many routing algorithms have been proposed in literature [2-4]. In the earlier research for distributed systems, it was assumed that wired systems had unlimited power. They had user interfaces, had fixed resources, treated each system node as important, and were location independent. WSNs in contrast are wireless systems with limited power, are constrained in energy consumption, and are real time, with dynamically varying resources $[5,6]$. Routing in WSN utilizing minimal energy has been proposed in the literature [7-11]. The power management solutions, at the software level, aim at reducing communications as broadcasting or listening to messages uses up energy. Minimizing message numbers cuts costs and a good MAC protocol ensures reduced collisions and retries. Better routing minimizes the number of messages sent by the use of short paths and congestion avoidance. Factors like efficient neighbor detection, localization, time synchronization, flooding, and query dissemination reduce the number of messages and increase the life of the network. There are varied solutions for scheduling sleep/wake-up patterns $[12,13]$ with most trying to keep up minimum nodes labeled sentries. The latter provides sensing coverage and allows others to sleep.

The clustering process divides a network into interconnected substructures, called clusters, with each cluster having many Sensor Node (SN) led by a Cluster Head (CH) which is the coordinator in this substructure [14] as seen in Figure 1. The $\mathrm{CH}$ is also a temporary base station which keeps in touch with other CHs. Nodes have four possible states: normal, isolated, cluster head, and gateway. Basically, nodes are in an 


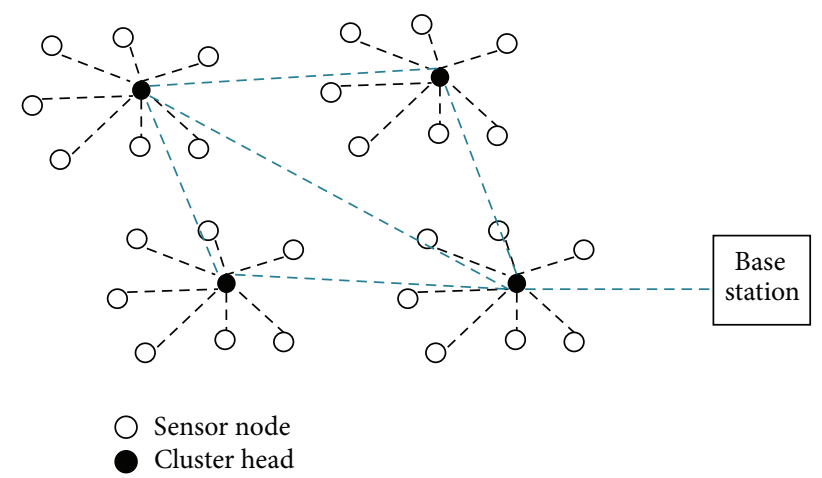

FIGURE 1: Block diagram of WSN deployment with cluster heads.

isolated state with each maintaining the neighbor table where neighbour node information is stored. Electing the $\mathrm{CH}$ is the basic step in clustering. Clustering is widely applied in WSN for managing power efficiently [15-17].

A clustering architecture in WSN environment enables features such as network scalability, communication overhead reduction, and fault tolerance. Cluster formation benefits routing as the cluster head and cluster gateways are responsible for the intercluster routing, thus, restricting, creating, and spreading of routing information. Local changes such as nodes changing cluster are updated only in the corresponding clusters and no update is required by the whole network. This significantly reduces information stored by each mobile node.

The major problem in WSNs is experienced by the sensor nodes nearest to the base station that require transmitting more number of packets than that of faraway nodes. Generally clustering algorithms use two methods for extending the lifetime. In the first method, the cluster heads with high residual energy are selected, and in the second method, to distribute the energy utilization between nodes in all clusters, the cluster heads are rotated periodically [18]. To choose the cluster heads, various techniques have been proposed in literature.

Other than clustering technique, tree based routing has been popularly used in WSN due to its energy efficiency [19-21]. Tree based techniques use the concept of selecting a root node before data transmission. A tree-like hierarchical path of nodes is constructed to connect the nodes. The WSN nodes construct a tree which can either be Minimum Spanning Tree (MST) or be optimal tree through which data is transmitted till it reaches the sink. The root node uses tree traversal algorithm to gather data about the children nodes. There are three methods in WSN tree routing protocols [19]. While the first does not create clusters the other two mix the clustering strategy with a tree routing algorithm. The latter strategy reduces low latency, while the tree routing algorithm improves energy efficiency $[22,23]$. In cluster based routing strategy, the sensor nodes in the network divide into several clusters. Each cluster then chooses a cluster head randomly or by cluster head election algorithm. The cluster head is responsible for gathering the data from the sensor nodes in its cluster. The aggregated data collected by the cluster head is then transmitted to the sink. Generally, a MST is created to connect the nodes and this reduces energy consumption either by transmitting packets through smaller distances or by reducing number of packets transmitted or by both techniques.

A modified Kruskal's Minimum Spanning Tree (MST) search algorithm based on distributed search by hierarchical clusters was proposed to search the network for small balanced weight routing spanning trees [5]. The proposed technique provided spanning trees with low maximum degree and larger diameter to balance energy consumption in WSN's routing. Based on energy matrix transmission the results proved that this approach extended WSN functional life by more than three times with respect to sensor transmission energy.

An energy efficient spanning tree (EESR) was proposed for multihop routing to increase the lifetime of the network [24]. The EESR provides location of the sensor nodes and base station and produces a sequence of routing paths consisting of suitable number of rounds. The results obtained by simulation reveal that the EESR method outperforms the other existing methods in relation to increasing the lifetime of the network.

A trajectory clustering technique was proposed for the purpose of selecting the cluster heads [25]. In this algorithm, the cluster heads are selected on the basis of traffic and they are periodically rotated. The cluster heads are selected using the trajectory based clustering technique and thus network lifetime is extended.

Guangyan et al. [26] proposed Dynamic Minimal Spanning Tree Routing Protocol (DMSTRP), an innovative cluster-based routing protocol, that enhanced Base Station Controlled Dynamic Clustering Protocol (BCDCP) by means of initiating MSTs rather than clubbing for the purpose of connecting nodes in clusters. When compared to LEACH and BCDCP, the DMSTRP performed well even in large network in terms of network lifetime and delay.

A distributed topology control technique was proposed in [27] to enhance energy efficiency and reduce radio interference in WSNs. Each network node makes local decisions about transmission power. These decisions conclude in a network topology preserving global connectivity. The fundamental control technique is the novel Smart Boundary Yao Gabriel Graph (SBYaoGG) and optimization ensures that all network links are symmetric and energy efficient. This technique was effective as compared to other approaches to topology control.

A combined algorithm (COM), a generalization of the MST and SPT, was proposed in [28] which dealt with the issue of executing the operation of Data Aggregation enhanced Convergecast (DAC) in an energy and latency efficient manner. The valuable portion of the total data gathered is approximated by assuming that each and every node in the network consists of a data item and a known application dependent data compression factor.

Multiple Cluster Heads Routing Protocol (MCHRP) [29] was proposed to address cluster head overload. This method improved LEACH by incorporating a decision function which is based on the cluster head's remaining energy, 
location, and frequency. The decision function selects the main cluster heads and the alternative cluster heads used for data acquisition, data fusion, and data transmission.

Cluster-based and Tree-based Power Efficient Data Collection and Aggregation (CTPEDCDA) protocol by Wang et al. [30] was based on clustering and MST to minimize energy consumption in WSN. MSTs are built by connecting the cluster heads to improve the transmission routing mechanism. Chhabra and Sharma [31] improved the power consumption by improving the first node death. This method combined both the cluster-based and the tree-based protocol to improve evenness of dissipated network energy.

Kumrai et al. [32] proposed evolutionary algorithm heuristically that optimizes the sensing coverage area and the installation cost in WSN by considering the sensor network connectivity as a constraint. The algorithm uses a population of individuals, each of which represents a set of wireless sensor nodes types and positions and evolves them via the proposed genetic operators. The proposed mutation and constraint-domination operators were designed to quickly seek the optimal solutions that meet the WSN installation requirements. Simulation result shows that the sensing coverage and installation cost were improved.

Karimi et al. [33] proposed two algorithms such as GP-Leach and HS-Leach. The energy consumption was improved by partitioning the network and using evolutionary algorithms for optimized cluster head selection considering WSN nodes position information and residual energy. The simulation results performed in MATLAB show that the proposed algorithms were more efficient and they increased the lifetime of network.

In this work, a Weighted Minimum Spanning Tree, Bee Algorithm-Simulated Annealing (BASA-WMST) algorithm is proposed. Cluster heads are selected based on the proposed optimization technique and WMST is used to find the shortest intrapath selection within the cluster. The proposed method computes the distance-based minimum spanning tree of the weighted graph for the multihop network. During route selection, the weights are dynamically adjusted based on the mobility, energy level, and distance of each sensor. Section 2 presents the problem formulation, Section 3 deals with the proposed methodology in detail, Section 4 shows the experimental results, and Section 5 concludes the paper.

\section{Problem Formulation}

Tree based routing has the advantage of lower control packet overheads but suffers from approximation error compared to cluster based routing. Cluster based routing provides better energy savings compared to tree based techniques. In this work it is proposed to combine the features of cluster based routing for cluster formation and cluster head selection and use minimum spanning tree for intracluster communication. Ideal clusters are formed when the network parameters like energy spent, lifetime, Packet Delivery Ratio, and end to end delay are optimized. Since most of the network parameters are additive in nature the optimization problem is NP hard. Several metaheuristic techniques including genetic algorithm have been proposed in literature. In this paper, bee algorithm in combination with simulated annealing was chosen due to its faster convergence and its capability to avoid local minima problem.

WSN network can be considered as a connected undirected graph represented by $G=(V, E)$, where $V$ is the vertices made up of $\left(v_{1}, \ldots, v_{n}\right)$ nodes and $E$ is the edges represented as $\left(e_{1,2}, e_{1,3}, \ldots, e_{i, j}, \ldots, e_{n-1, n}\right)$ the connection between nodes [34]. In this work the normalized values of mobility, delay, and remaining energy are represented on the edges. Each edge may then be defined by the attributes represented in positive real numbers and denoted by $w_{i, j}=$ $w_{i, j}^{1}, w_{i, j}^{2}, \ldots, w_{i, j}^{m}$.

Let $x=x_{1,2}, x_{1,3}, \ldots, x_{i, j}, \ldots, x_{n-1, n}$ be defined as the connectivity between node $i$ and $j$ :

$$
x_{i, j}= \begin{cases}1 & \text { if } e_{i, j}=1 \text { and is selected } \\ 0 & \text { otherwise }\end{cases}
$$

The proposed technique can be formulated as in

$$
\begin{aligned}
\min f_{1}(x) & =\sum w_{i, j}^{1} x_{i, j} \\
\min f_{2}(x) & =\sum w_{i, j}^{2} x_{i, j} \\
\vdots & \\
\min f_{m}(x) & =\sum w_{i, j}^{m} x_{i, j},
\end{aligned}
$$

where $f_{i}(x)$ is the objective to be minimized for the problem, $i=1, \ldots, n-1 ; j=1, \ldots, n$ subject to $x \in X$. These objectives either can be formulated as a multiobjective function or can be represented as in

$$
\min f_{i}(x)=\alpha \sum w_{i, j}^{1} x_{i, j}+\beta \sum w_{i, j}^{2} x_{i, j}+\cdots,
$$

where $\alpha+\beta+\cdots=1$.

Since node mobility, delay, and remaining energy are used as the edge in the graph, the objective function can be formulated as in

$$
\begin{aligned}
\min f_{i}(x)= & \alpha(\min (\operatorname{mobility}))+\beta(\min (\text { delay })) \\
& +\gamma(\min (1-\text { remaining_energy })) .
\end{aligned}
$$

The following assumptions are made for the sensor network.

(1) Nodes are dispersed randomly.

(2) The energy of sensor nodes is limited and uniform initially.

(3) Nodes are location unaware.

(4) The transmitting power of the nodes varies depending on the distance to the receiver.

(5) Approximate distance is estimated based on the received signal strength. 


\section{Methodology}

The node energy model is based on [35]. The energy dissipated to transmit $n$ bit is given in

$$
\begin{aligned}
& E_{\text {diss_Tx }} \\
& \begin{array}{l}
=n(\text { Energy_dissipated_tranmitter_electronics } \\
+(\text { Energy_dissipated_transmitter_amplifier } \\
\quad * \text { distance_squared })) .
\end{array}
\end{aligned}
$$

The energy dissipated to receive $n$ bit is given in

$$
\left.E_{\text {diss_Rx }}=n \text { (Energy_dissipated_receiver_electronics }\right) \text {. }
$$

Power consumed for a given time period $t$ can be computed by dividing the dissipated energy by time and is given by

$$
\frac{E_{\text {diss_Rx }}+E_{\text {diss_Tx }}}{t} .
$$

The mobility of a node is estimated using the Free Space Path Loss (FSPL) model. The relation between FSPL, frequency of radio signal, and distance between the transmitter and receiver is given by

$$
\operatorname{FSPL}(\mathrm{dB})=20 \log (d)+20 \log (f)+k,
$$

where $d$ is the distance, $f$ is the frequency, and log is the logarithm to base $10 . K$ is a constant and is equal to 32.44 when frequency is measured in Mhz and distance is measured in Kilometer. Another method to compute the FSPL is using the fade margin and it is given by

$$
\begin{aligned}
\text { FSPL }= & \text { Energy_dissipated_Tx_electronics } \\
& + \text { Energy_dissipated_Tx_amplifier } \\
& + \text { Energy_dissipated_Rx_electronics. }
\end{aligned}
$$

Using the two FSPL equations (8) and (9), the distance can be computed by

$$
d=\frac{10(\text { Free Space Path Loss }-32.44-20 \log (f))}{20} .
$$

To find the distance travelled by nodes $i$ and $j$ with respect to each other during time $n$, the distance between the nodes is computed at time $t$ and $t=n$ if high mobility increases the reclustering process and increases the energy consumption. The objective is to form clusters based on low mobility which leads to lower energy consumption and lower delays due to lower link breakages. The mobility of the node can be computed by

$$
m=\frac{d_{t}-d_{t+n}}{D}\left\{\begin{array}{l}
>0.5 \text { implies high mobility } \\
\leq 0.5 \text { implies normal mobility } \\
0 \text { implies no mobility } \\
<0 \text { implies nodes converging }
\end{array}\right\} .
$$

TABLE 1: Information maintained in the neighborhood table.

\begin{tabular}{ll}
\hline Parameter & Description \\
\hline$V_{i}$ & Node $i$ \\
$V_{j}$ & A neighbor node in cluster range of $V_{i}$ \\
$\mathrm{RE}_{V_{i}}$ & Residual energy of $V_{i}$ \\
$\mathrm{Dis}_{V_{j}}$ & Distance between $V_{i}$ and $V_{j}$ \\
$\mathrm{RE}_{V_{j}}$ & Residual energy of $V_{j}$ \\
Ech_Msg & Elect cluster head message \\
Crt_Msg & Create tree message \\
\hline
\end{tabular}

Each node stores in its neighborhood table the information about its neighbors, as shown in Table 1. Each node broadcasts the Ech_Msg, at the beginning of each round which contains residual energies, within radio range $r$. All nodes within the cluster range of one node are considered as the neighbors of this node. On receiving the Ech_Msg nodes update the neighborhood table.

The flow chart of the proposed technique is shown in Figure 2. Bee algorithm-simulated annealing algorithm is proposed to avoid the local minima problem faced by bee algorithm and to select the best cluster heads by forming ideal clusters. Clustering is achieved by dividing arbitrarily organized sensors into the best possible number of selfdetermining clusters with cluster head and optimal route to form the initial population. The edge weights between nodes are computed and the objective function is computed. These initial solutions become the initial food source in the proposed BASA algorithm. Once the initial population is found, the bee algorithm is initiated. Each node broadcasts its ID along with its weight $W_{i}$ to the neighboring nodes and stores the weights $W_{j}$ of the other nodes within its transmission range.

Bee's algorithm is a population-based search algorithm inspired by bees foraging behaviour [36]. The algorithm starts with search space being populated by worker bees being placed randomly at the location of the initial food source. The fitness of sites visited by worker bees is evaluated and bees with the best fitness continue to be worker bees. Bees which have visited sites with lower fitness value are delegated to onlooker bees. The location of food source with the best fitness becomes the new search location for better solutions. Effectively a cluster with $\mathrm{CH}$ can either add new nodes to increase the cluster or remove some nodes to decrease the cluster. Similarly, the $\mathrm{CH}$ can be rotated within the cluster. This is achieved by searching neighborhoods of selected sites by assigning scout bees to search near the best $e$ sites. Neighborhood searches of the best $e$ sites are made detailed by recruiting more bees other than the selected bees to follow them. This differential recruitment along with scouting is a key bee's algorithm operation.

The probability $p_{i}$ of selecting a food source $i$ can be determined by using

$$
p_{i}=\frac{\text { fit }_{i}}{\sum_{n=1}^{\mathrm{SN}} \mathrm{fit}_{n}}
$$




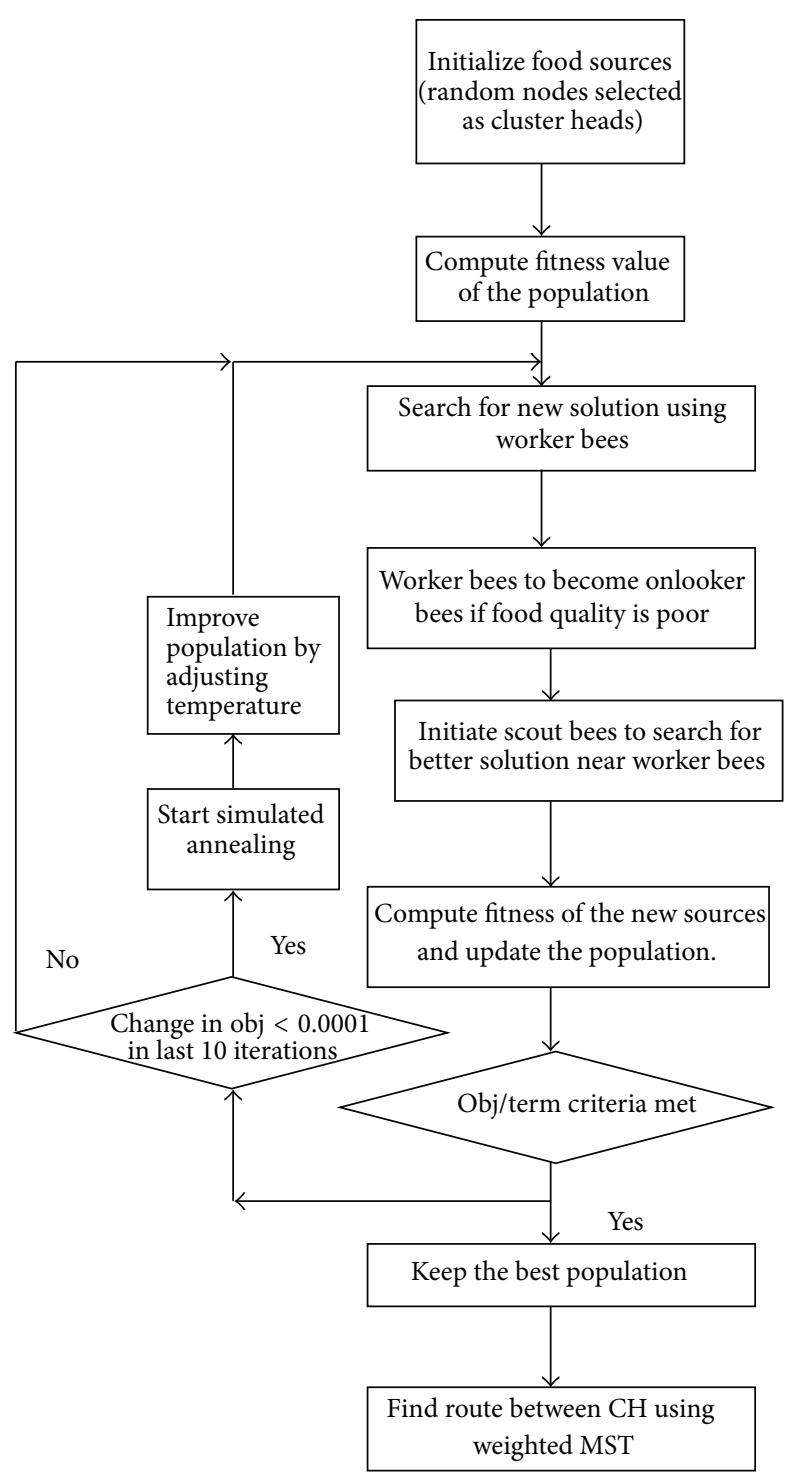

FIGURE 2: Flowchart of the proposed technique.

and the fitness is given as in

$$
\text { fitness }(i)= \begin{cases}1+\operatorname{abs}(f(i)), & f(i)<0 \\ \frac{1}{(f(i))}+1, & f(i) \geq 0\end{cases}
$$

where fit $_{i}$ is the fitness value of $i$ th solution which represents the nectar amount at food source at $i$ th position and $\mathrm{SN}$ is the number of employed bees and also a number of food sources.

The process is iterated till the termination criteria are reached or till the improvement in the fitness does not increase by more than 0.001 in the last 10 iterations. If there is no improvement in the solution, the algorithm could have struck in the local minima. Simulated annealing is starting to climb out of the local minima problem.

The simulated annealing (SA) was introduced in 1983 which is based on the ideas formulated in the early 1950s [37]. Simulated annealing is a relatively straight forward

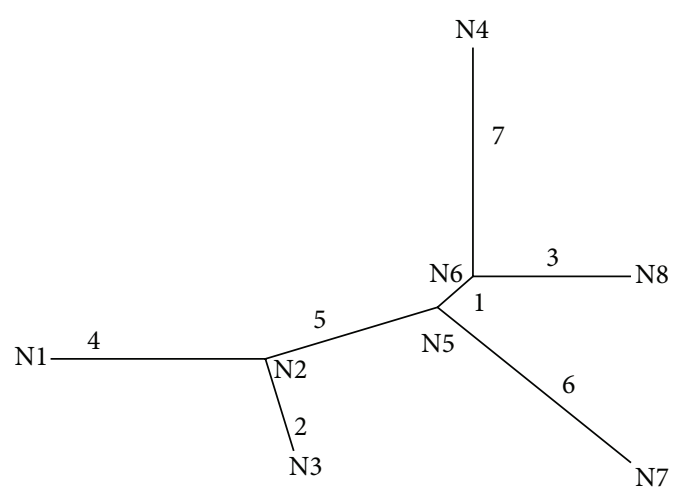

FIGURE 3: A tree connecting eight vertices.

algorithm which includes metropolis Monte Carlo method. The metropolis Monte Carlo algorithm is well suited for simulated annealing, since only energetically feasible states can be sampled at any given temperature. Therefore the simulated annealing algorithm starts at a high temperature with simulation of metropolis Monte Carlo algorithm. The temperature is slowly reduced such that the search space becomes smaller for the metropolis simulation, and when the temperature is low then the system has hopefully settled into the most favorable state. Simulated annealing can also be used for searching the optimum solution of the problems by properly determining the initial (high) and final (low) effective temperatures which are used in place of $k T$ (where $k$ is a Boltzmann's constant) in the acceptance checking and deciding what constitutes a Monte Carlo step [38].

Simulated annealing is a probabilistic method [39] to find global minimum of a cost function that can have several local minima. Simulated annealing emulates the physical process wherein a solid is slowly cooled so that when eventually its structure is frozen, this happens at a minimum energy configuration. Simulated annealing to compute the probability of acceptance:

$$
P_{a}= \begin{cases}e^{-k \Delta E / T} & \Delta E>0 \\ 1 & \Delta E \leq 0,\end{cases}
$$

where $\Delta E$ is the difference between the solution error after it has perturbed and the solution error before it was perturbed, $T$ is the current temperature, and $k$ is a suitable constant.

On identifying the potential cluster heads, the MST algorithm is used for tree construction to find the intracluster routes. Suppose that $n$ points are given in different dimensions, then a tree spanning to these points is a set of straight line segments joining pairs of points [40], so that (1) there are no closed loops, (2) a line visits each point at least once, and (3) the tree is connected.

Figure 3 shows an example of a tree of integer segment lengths. If, for example, vertices N3 and N7 are joined, a closed loop is formed and the result would not be a tree. The length of a tree is the sum of its segments lengths. When a set of $n$ points and the lengths of all $(n / 2)$ segments are given, a spanning tree of minimum length (MST) is required. The MST is computed using reaching the base station. 
TABLE 2: The parameters of the network simulation.

\begin{tabular}{lc}
\hline Parameters & Values \\
\hline $\begin{array}{l}\text { Initial energy of nodes } E_{\text {init }} \\
\text { Amplification coefficient of the free space }\end{array}$ & $0.6 \mathrm{~J}$ \\
model $E_{f s}$ & $10 \mathrm{pJ}-\mathrm{m}^{2} / \mathrm{b}$ \\
$\begin{array}{l}\text { Amplification coefficient of the multipath } \\
\text { transmission model } E_{\text {amp }}\end{array}$ & $0.0025 \mathrm{pJ}-\mathrm{m}^{2} / \mathrm{b}$ \\
Circuit loss $E_{\text {elec }}$ & $50 \mathrm{~nJ} / \mathrm{b}$ \\
$\begin{array}{l}\text { Data packet length } \\
\text { Control packet length }\end{array}$ & $8000 \mathrm{~b}$ \\
$\begin{array}{l}\text { Initial number of worker bees } \\
\text { Food source-the initial search space for }\end{array}$ & $120 \mathrm{~b}$ \\
cluster head (random) & 20 \\
Encoding & 20 \\
Termination condition & Binary with \\
\hline
\end{tabular}

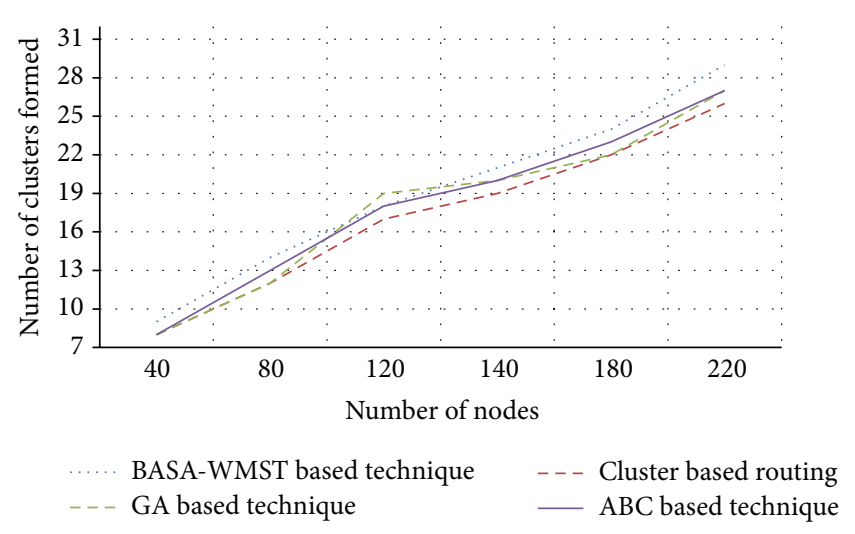

FIGURE 4: Number of clusters formed.

With traditional MST algorithms, construction cost of a minimum spanning tree is $O(m \log n)$, where $m$ is the number of graph edges and $n$ is the number of vertices [41]. The weight of a tree edge is computed by Euclidean distance between two end points. The average weight $w$ of MST edges based on remaining energy and delay is computed. Any edge with a weight $w>w_{\text {avg }}$ is removed leading to a set of disjoint subtrees $t=\left\{t_{1}, t_{2}, \ldots t_{i}\right\}$.

The so formed routes are optimal from the spatial perspective since the cluster heads are uniformly distributed over the imperfectly formed wireless sensor network.

\section{Experimental Set-Up and Results}

Experiments were conducted with different number of mobile sensor nodes, spread over an area of $1000 \mathrm{~m}$ by $1000 \mathrm{~m}$ with the Base Station being stationary at location $(500,500)$. The simulation parameters and bee algorithm parameters are shown in Table 2.

Experiments were conducted to simulate the proposed technique and are compared to cluster based routing, GA based cluster formation, and ABC based cluster optimization. Figure 4 shows the number of clusters formed. The proposed BASA WMST technique increases the average number of
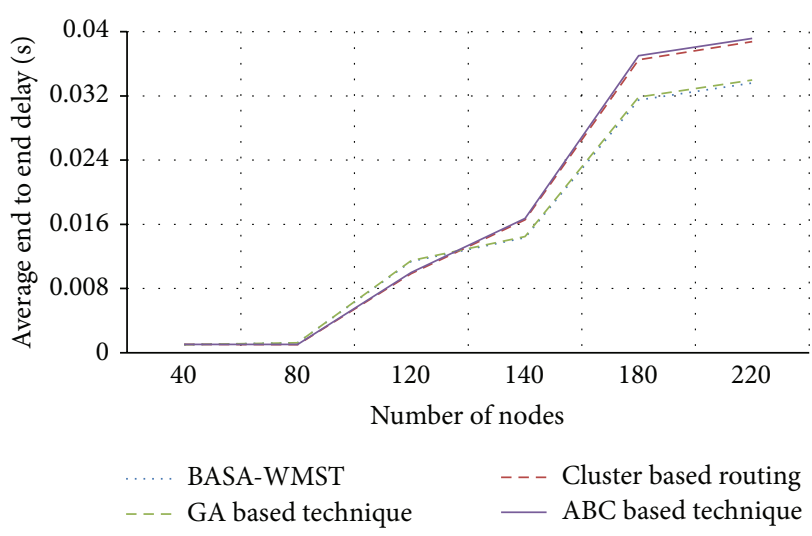

Figure 5: Average end to end delay.

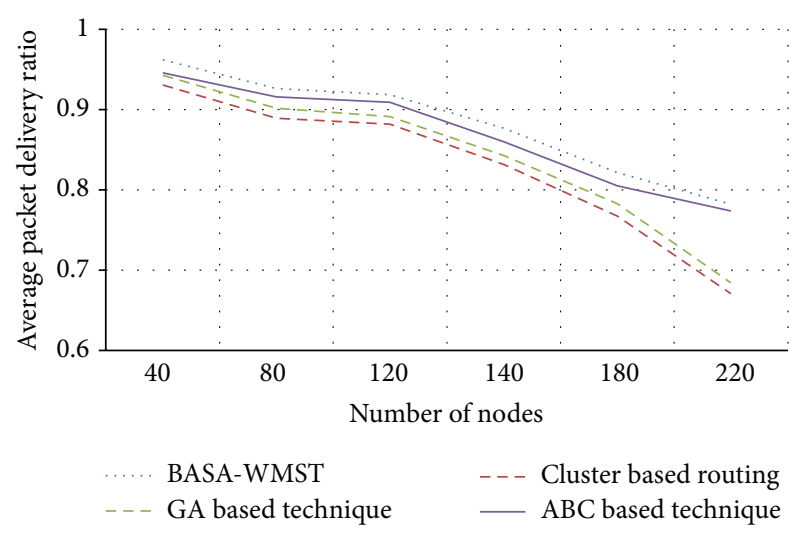

Figure 6: Average Packet Delivery Ratio.

clusters across by $10.41 \%$ compared to cluster based routing for varying number of nodes in the network. ABC produces better clusters compared to GA.

Figure 5 shows the average end to end delay obtained in the network for different number of nodes. As the number of nodes increases, the performance improvement of the proposed technique is in par with GA based technique showing a small marginal average improvement of $1.27 \%$ compared to GA based technique.

However, both GA and proposed BASA-WMST show significant decrease in end to end delay as the number of nodes is increased. End to end delay decreased over $14 \%$ when compared to cluster based technique. This becomes significant for WSN used in streaming applications. Figure 6 shows the Packet Delivery Ratio obtained under different number of nodes.

The average PDR in the proposed BASA WMST improved by $6.49 \%$ when compared to cluster based routing for various numbers of nodes in the network. Compared to GA the PDR improvement was significant by $4.98 \%$ and by $1.47 \%$ compared to ABC based technique. Figure 7 shows the life time of the network. $\mathrm{ABC}$ and the proposed technique significantly improve the life of the network compared to GA based technique. 

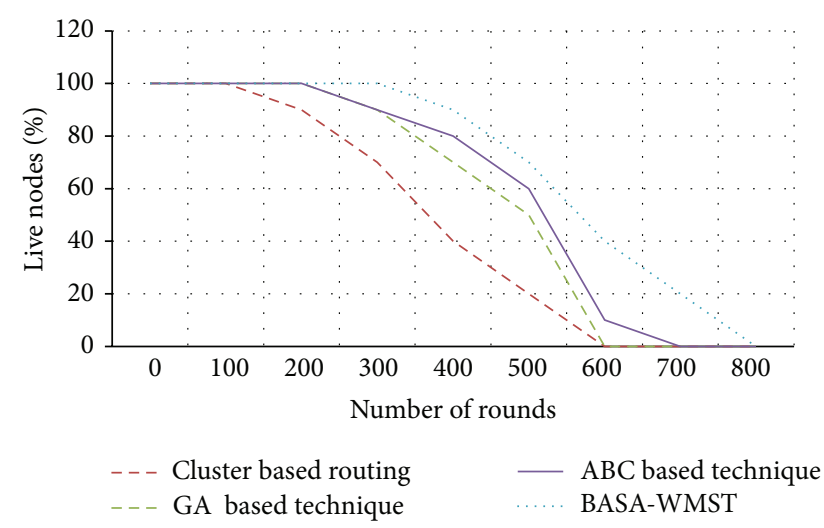

FIGURE 7: Network life time.

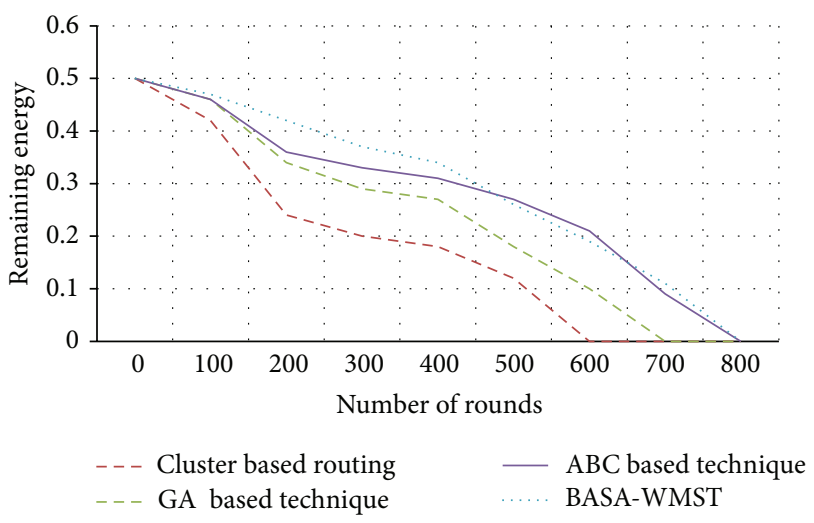

Figure 8: Remaining energy.

Figure 8 shows the average remaining energy in the nodes. The energy savings are significant in the proposed technique compared to GA based technique. Average energy to the tune of $21.67 \%$ is observed compared to GA.

\section{Conclusion}

WSN routing protocols must perform efficiently under mobility and energy constraints. In this paper, clustering is achieved through a hybrid algorithm that divides arbitrarily organized sensors into the best possible number of selfdetermining clusters with cluster head and optimal route to base station using a novel optimization BASA-WMST. Bee algorithm is incorporated to increase the information exchange among bees and SA is used to escape local optima. Intracluster route is selected from the optimal trees based on weights described. The proposed routing was simulated and compared with conventional cluster based routing and other optimization techniques showing improvements in the QOS.

\section{Conflict of Interests}

The authors declare that there is no conflict of interests regarding the publication of this paper.

\section{References}

[1] S. C. Misra and I. Woungang, Guide to Wireless Sensor Networks, vol. 7, Springer, New York, NY, USA, 2009.

[2] C. K. Toh, A. N. Le, and Y. Z. Cho, "Load balanced routing protocols for ad hoc mobile wireless networks," IEEE Communications Magazine, vol. 47, no. 8, pp. 78-84, 2009.

[3] J. Le, J. C. Lui, and D. M. Chiu, "DCAR: distributed codingaware routing in wireless networks," IEEE Transactions on Mobile Computing, vol. 9, no. 4, pp. 596-608, 2010.

[4] N. S. Moayedian and S. J. Golestani, "Optimal scheduling and routing in wireless networks: a new approach," in Proceedings of the IEEE Wireless Communications and Networking Conference (WCNC '09), pp. 1-6, IEEE, April 2009.

[5] A. Gagarin, S. Hussain, and L. T. Yang, "Distributed search for balanced energy consumption spanning trees in wireless sensor networks," in Proceedings of the International Conference on Advanced Information Networking and Applications Workshops (WAINA '09), pp. 1037-1042, Bradford, UK, May 2009.

[6] J. N. Al-Karaki and A. E. Kamal, "Routing techniques in wireless sensor networks: a survey," IEEE Wireless Communications, vol. 11, no. 6, pp. 6-28, 2004.

[7] T. Watteyne, A. Molinaro, M. G. Richichi, and M. Dohler, "From MANET to IETF ROLL standardization: a paradigm shift in WSN routing protocols," IEEE Communications Surveys and Tutorials, vol. 13, no. 4, pp. 688-707, 2011.

[8] B. Priya and S. S. Manohar, "EE-MAC: energy efficient hybrid MAC for WSN," International Journal of Distributed Sensor Networks, vol. 2013, Article ID 526383, 9 pages, 2013.

[9] E. Ahvar, S. Ahvar, G. M. Lee, and N. Crespi, "An energy-aware routing protocol for query-based applications in wireless sensor networks," The Scientific World Journal, vol. 2014, Article ID 359897, 9 pages, 2014.

[10] X. Zhu, L. Shen, and T. P. Yum, "Hausdorff clustering and minimum energy routing for wireless sensor networks," IEEE Transactions on Vehicular Technology, vol. 58, no. 2, pp. 990997, 2009.

[11] T. W. Kuo and M. J. Tsai, "On the construction of data aggregation tree with minimum energy cost in wireless sensor networks: NP-completeness and approximation algorithms," in Proceedings of the IEEE Conference on Computer Communications (INFOCOM '12), pp. 2591-2595, IEEE, March 2012.

[12] O. Khader, A. Willig, and A. Wolisz, "Distributed wakeup scheduling scheme for supporting periodic traffic in WSNs," in Proceedings of the Wireless Conference, pp. 287-292, IEEE, May 2009.

[13] U. Jang, S. Lee, and S. Yoo, "Optimal wake-up scheduling of data gathering trees for wireless sensor networks," Journal of Parallel and Distributed Computing, vol. 72, no. 4, pp. 536-546, 2012.

[14] S. Bandyopadhyay and E. J. Coyle, "An energy efficient hierarchical clustering algorithm for wireless sensor networks," in Proceedings of the 22nd Annual Joint Conference on the IEEE Computer and Communications Societies, vol. 3, pp. 1713-1723, IEEE Societies, April 2003.

[15] T. Kwon and M. Gerla, "Clustering with power control," in Proceedings of the IEEE Military Communications Conference (MILCOM '99), vol. 2, pp. 1424-1428, November 1999.

[16] D. De, A. Sen, and M. D. Gupta, "Cluster based energy efficient lifetime improvement mechanism for WSN with multiple mobile sink and single static sink," in Proceedings of the 3rd International Conference on Computer and Communication Technology (ICCCT '12), pp. 197-199, IEEE, November 2012. 
[17] M. J. Reddy, P. S. Prakash, and P. C. Reddy, "Homogeneous and heterogeneous energy schemes for hierarchical cluster based routing protocols in WSN: a survey,' in Proceedings of the 3rd International Conference on Trends in Information, Telecommunication and Computing, pp. 591-595, Springer, New York, NY, USA, 2013.

[18] G. Ma and Z. Tao, "A hybrid energy- and time-driven cluster head rotation strategy for distributed wireless sensor networks," International Journal of Distributed Sensor Networks, vol. 2013, Article ID 109307, 13 pages, 2013.

[19] G. Zheng and Z. Hu, "Tree routing protocol with location-based uniformly clustering strategy in WSNs," Journal of Networks, vol. 5, no. 11, pp. 1373-1380, 2010.

[20] J. Zhang, Y. Xie, D. Liu, and Z. Zhang, "OCTBR: optimized clustering tree based routing protocol for wireless sensor networks," in Internet of Things, pp. 192-199, Springer, Berlin, Germany, 2012.

[21] X. Wang and H. Qian, "Constructing a 6LoWPAN wireless sensor network based on a cluster tree," IEEE Transactions on Vehicular Technology, vol. 61, no. 3, pp. 1398-1405, 2012.

[22] S. S. Satapathy and N. Sarma, "TREEPSI: tree based energy efficient protocol for sensor information," in Proceeding of the IFIP International Conference on Wireless and Optical Communications Networks, Bangalore, India, April 2006.

[23] D. Singhal, S. Barjatiya, and G. Ramamurthy, "A novel network architecture for cognitive wireless sensor network," in Proceedings of the International Conference on Signal Processing, Communication, Computing and Networking Technologies (ICSCCN '11), pp. 76-80, IEEE, July 2011.

[24] S. Hussain and O. Islam, "An energy efficient spanning tree based multi-hop routing in wireless sensor networks," in Proceedings of the IEEE Wireless Communications and Networking Conference (WCNC '07), pp. 4383-4388, Kowloon, Hong Kong, 2007.

[25] M. Hazarath, I. Lucio, and C. Luca, "CAST—a novel trajectory clustering and visualization tool for spatio temporal data," in Proceedings of the 1st International conference on Intelligent Human Computer Interaction (IHCI '09), pp. 169-175, Springer, 2009.

[26] H. Guangyan, L. Xiaowei, and H. Jing, "Dynamic minimal spanning tree routing protocol for large wireless sensor networks," in Proceedings of the 1st IEEE Conference on Industrial Electronics and Applications (ICIEA '06), pp. 1-5, Singapore, May 2006.

[27] T. M. Chiwewe and G. P. Hancke, "A distributed topology control technique for low interference and energy efficiency in wireless sensor networks," IEEE Transactions on Industrial Informatics, vol. 8, no. 1, pp. 11-19, 2011.

[28] S. Upadhyayula and S. K. S. Gupta, "Spanning tree based algorithms for low latency and energy efficient data aggregation enhanced convergecast (DAC) in wireless sensor networks," Ad Hoc Networks, vol. 5, no. 5, pp. 626-648, 2007.

[29] D. Tang, X. Liu, Y. Jiao, and Q. Yue, "A load balanced multiple Cluster-heads routing protocol for wireless sensor networks," in Proceedings of the IEEE 13th International Conference on Communication Technology (ICCT '11), pp. 656-660, Jinan, China, September 2011.

[30] W. Wang, B. Wang, Z. Liu, L. Guo, and W. Xiong, "A clusterbased and tree-based power efficient data collection and aggregation protocol for wireless sensor networks," Information Technology Journal, vol. 10, no. 3, pp. 557-564, 2011.
[31] G. S. Chhabra and D. Sharma, "Cluster-tree based data gathering in wireless sensor network," International Journal of Soft Computing and Engineering, vol. 1, no. 1, pp. 27-31, 2011.

[32] T. Kumrai, P. Champrasert, and R. Kuawattanaphan, "Heterogeneous wireless sensor network (WSN) installation using novel genetic operators in a multiobjective optimization evolutionary algorithm," in Proceedings of the 9th International Conference on Natural Computation (ICNC '13), pp. 606-611, IEEE, 2013.

[33] M. Karimi, H. R. Naji, and S. Golestani, "Optimizing clusterhead selection in wireless sensor networks using genetic algorithm and harmony search algorithm," in Proceedings of the 20th Iranian Conference on Electrical Engineering (ICEE '12), pp. 706710, IEEE, Tehran, Iran, May 2012.

[34] W. Guo, B. Zhang, G. Chen, X. Wang, and N. Xiong, "A PSOoptimized minimum spanning tree-based topology control scheme for wireless sensor networks," International Journal of Distributed Sensor Networks, vol. 2013, Article ID 985410, 14 pages, 2013.

[35] W. R. Heinzelman, A. Chandrakasan, and H. Balakrishnan, "Energy-efficient communication protocol for wireless microsensor networks," in Proceedings of the 33rd Annual Hawaii International Conference on System Siences (HICSS '00), p. 10, January 2000.

[36] D. T. Pham, A. Ghanbarzadeh, E. Koc, S. Otri, S. Rahim, and M. Zaidi, "The bees algorithm-a novel tool for complex optimisation problems," in Proceedings of the 2nd Virtual International Conference on Intelligent Production Machines and Systems (IPROMS '06), pp. 454-459, 2006.

[37] M. Kumar and D. R. Gupta, "A comparative study using simulated annealing and fast output sampling feedback technique based pss design for single machine infinite bus system modeling," International Journal of Engineering Research and Applications, vol. 2, no. 2, pp. 223-228, 2012.

[38] A. Tamilarasi, "An enhanced genetic algorithm with simulated a nnealing for job-shop scheduling," International Journal of Engineering, Science and Technology, vol. 2, no. 1, pp. 144-151, 2010.

[39] S. Kirkpatrick, C. D. Gelatt, and M. P. Vecchi, "Optimization by simulated annealing," Science, vol. 220, no. 4598, pp. 671-680, 1983.

[40] J. C. Gower and G. J. S. Ross, "Minimum spanning trees and single linkage cluster analysis," Applied Statistics, vol. 18, pp. 5464, 1969.

[41] D. R. Edla and P. K. Jana, "Minimum spanning tree based clustering using partitional approach," in Proceedings of the International Conference on Frontiers of Intelligent Computing: Theory and Applications (FICTA '13), pp. 237-244, Springer, Berlin, Germany, 2013. 


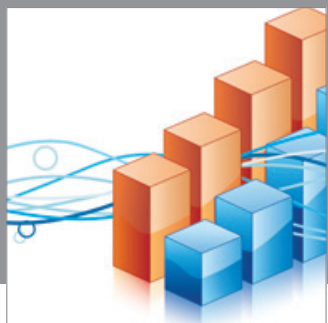

Advances in

Operations Research

mansans

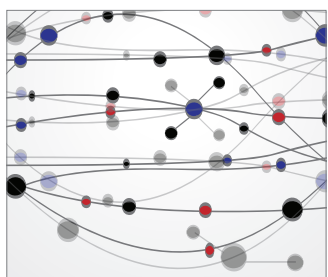

The Scientific World Journal
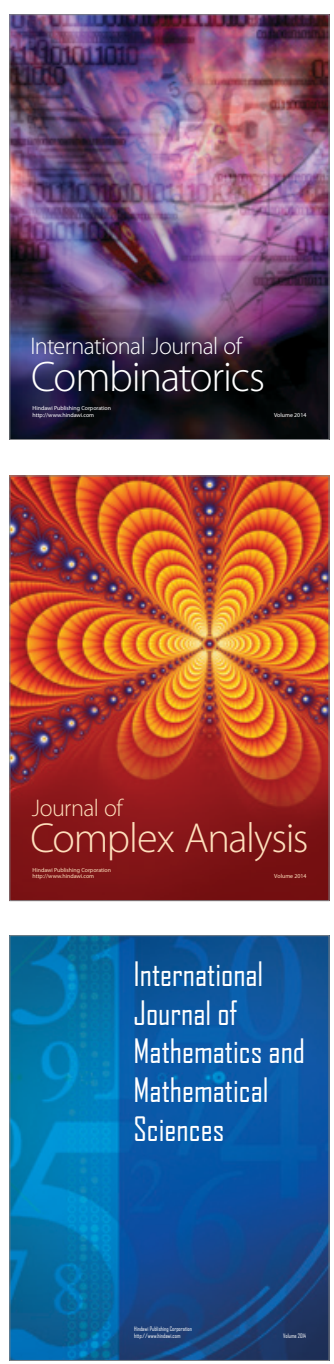
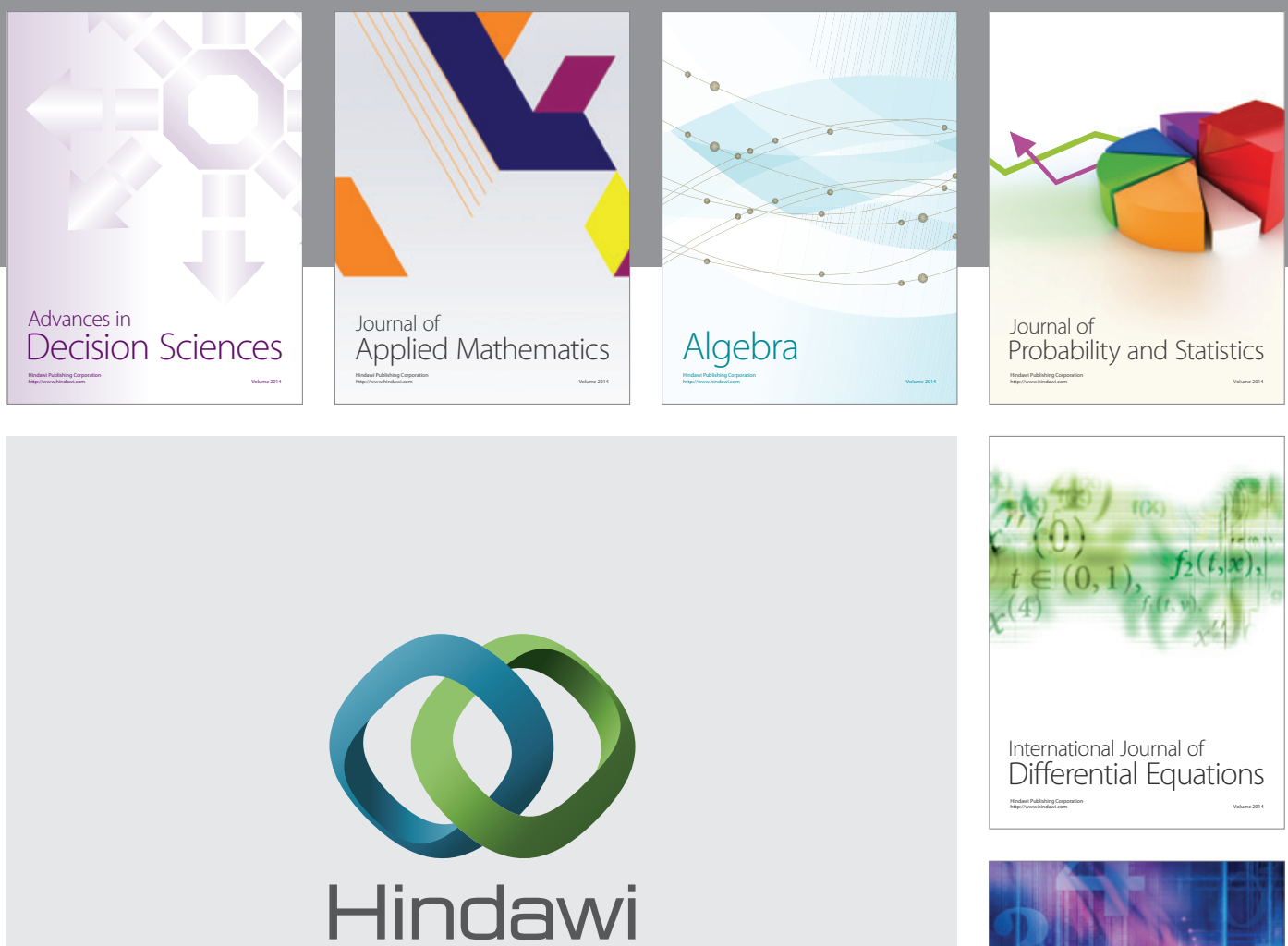

Submit your manuscripts at http://www.hindawi.com
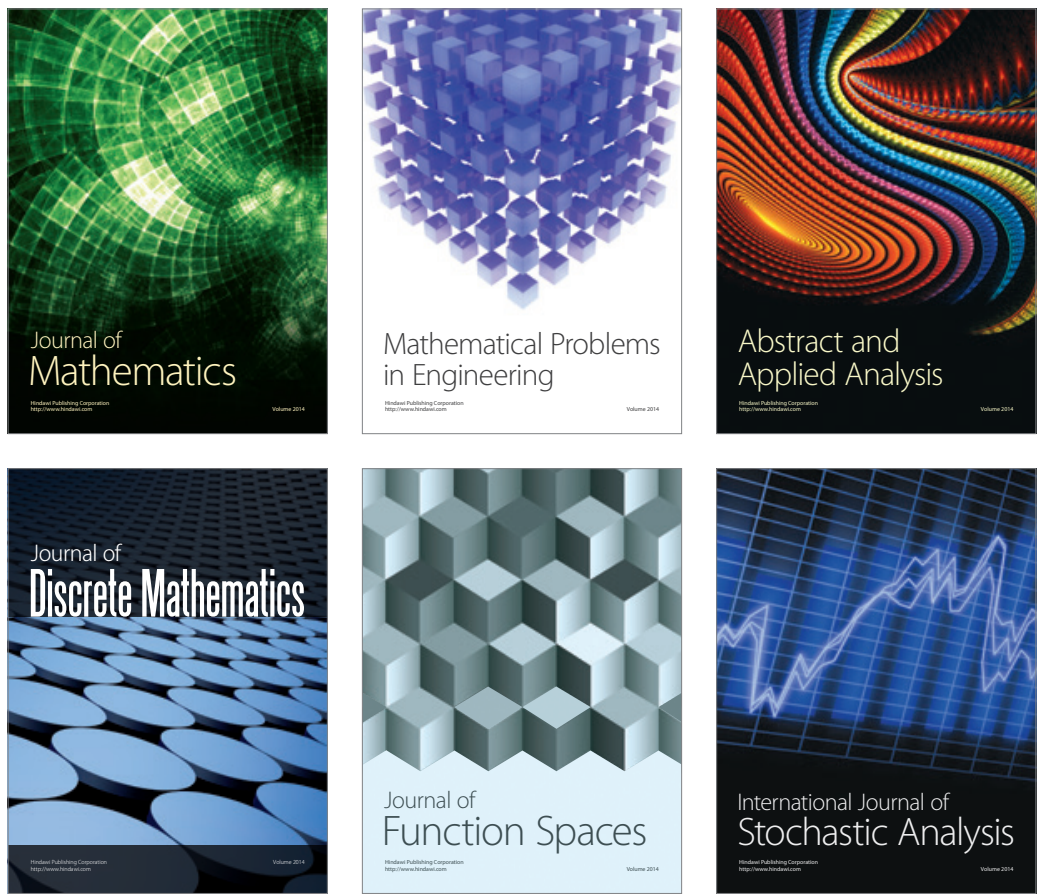

Journal of

Function Spaces

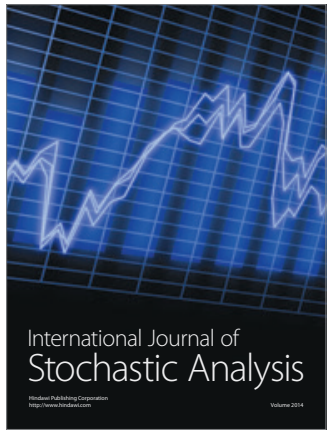

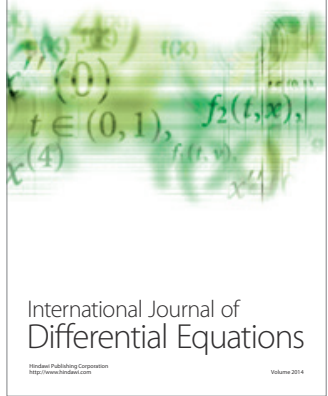
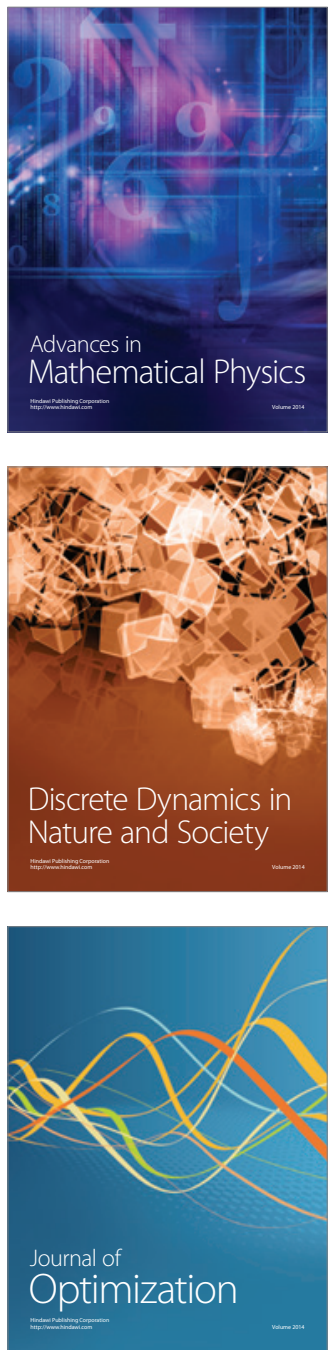